\title{
Kimchi and Asian fermented vegetables: candidates for the partial control of the COVID-19 pandemic
}

\author{
Jean Bousquet ${ }^{1}$, Josep Anto ${ }^{2}$, Wienczyslawa Czarlewski ${ }^{3}$, Tari Haahtela ${ }^{4}$, Susana Fonseca ${ }^{5}$, \\ Guido Iaccarino ${ }^{6}$, Hubert Blain ${ }^{7}$, Hae-Sim Park ${ }^{8}$, Aziz Sheikh ${ }^{9}$, Cezmi Akdis ${ }^{10}$, and \\ Torsten Zuberbier ${ }^{11}$ \\ ${ }^{1}$ Université Versailles, St-Quentin-en-Yvelines \\ ${ }^{2}$ ISGLoBAL \\ ${ }^{3}$ Medical Consulting \\ ${ }^{4}$ Helsinki University Hospital \\ ${ }^{5}$ GreenUPorto - Sustainable Agrifood Production Research Centre \\ ${ }^{6}$ Federico II University Hospital \\ ${ }^{7} \mathrm{CHU}$ Montpellier \\ ${ }^{8}$ Ajou University School of Medicine \\ ${ }^{9}$ University of Edinburgh \\ ${ }^{10}$ University of Zurich \\ ${ }^{11}$ Charité Universitätsmedizin Berlin
}

July 28, 2020

To the Editor,

A COVID-19 epidemic started in China and then disseminated to other Asian countries before becoming a pandemic. It appears that the pandemic has so far resulted in proportionately fewer deaths in China and most Eastern Asian countries. Many reasons can explain this picture. ${ }^{1}$ One of them is the type of diet in the low mortality countries. ${ }^{2}$

This paper is the sixth of a series attempting to understand the role of diet in the differences of COVID-19 death rates between and within countries with the aim to identify potential preventive measures against COVID-19. The concept paper ${ }^{2}$ was followed by two ecological studies comparing death rates in European countries and the consumption of vegetables or fermented foods. ${ }^{3,4}$ We then proposed that sulforaphane from cruciferous vegetables ${ }^{1}$ and lactobacilli from fermented foods (submitted) were possibly involved in the reduction of insulin resistance in COVID-19.

It is noteworthy that fermented foods are largely used in Asia. ${ }^{5,6}$ It is therefore important to check whether some commonly eaten fermented foods in these countries may explain geographic differences in COVID-19. Kimchi will be used as a model of fermented cabbage.

\section{Geographic differences in COVID-19 death rates}

When comparing death rates, large differences exist between and within countries and the evolution of the pandemic differs largely between countries (Figure 1). As the temporal dynamic of the pandemic may have changed the geographical patterns observed in our previous analyses, ${ }^{2}$ we focus on the more recent mortality trends.

Although there are many pitfalls in analyzing death rates for COVID- $19,{ }^{2}$ the evolution of death rates 
between May 20 and July 18 shows a dramatic increase in Latin America and some increase in European countries, certain African countries, the Middle East, India, Pakistan and some of the South East Asian countries. However, there is no change in the very low death rates of Cambodia, China, Japan, Korea, Lao, Malaysia, Taiwan, Vietnam as well as of many Sub-Saharan African countries, Australia and New Zealand. This geographical pattern is very unlikely to be totally due to reporting differences between countries.

\section{Ecological studies on food consumption and death rates in countries}

Although many factors are involved in COVID-19 mortality, ${ }^{7-13}$ a recent hypothesis proposed that differences between countries may partly be explained by diet with fermented foods and certain vegetables that are potent anti-oxidants. ${ }^{2}$ Two studies examined the association between the COVID-19 mortality rate in EU countries and diet. The consumption of fermented vegetables, head cabbage and cucumber was associated with lower death rates. ${ }^{3,4}$ Even though these are ecological studies, they support the association between diet and fatality. A healthy diet, rich in antioxidants, has been proposed as a therapeutic strategy to mitigate the cytokine storm that develops in COVID-19. ${ }^{14}$

\section{Angiotensin-converting enzyme 2 (ACE2) and COVID-19}

COVID-19 is more severe in older adults and/or patients with comorbidities, such as diabetes, obesity, kidney disease or hypertension, suggesting a role for insulin resistance. ${ }^{15}$ Although differences exist between countries, the same risk factors for severity were found globally ${ }^{16-20}$, suggesting common mechanisms. Moreover, the severe outcomes of COVID-19 - including lung damage, cytokine storm or endothelial damage - appear to exist globally, again suggesting common mechanisms.

The angiotensin-converting enzyme 2 (ACE-2) is part of the dual system, the renin-angiotensin-system (RAS), including the ACE-Angiotensin-II-AT ${ }_{1} \mathrm{R}$ axis. $\mathrm{AT}_{1} \mathrm{R}$ is involved in most Angiotensin II effects, including oxidative stress generation, pro-inflammatory, pro-fibrotic effects in the respiratory system, endothelial damage and insulin resistance. ${ }^{21,22}$ SARS-CoV-2 binds to and downregulates ACE-2, enhancing the $\mathrm{AT}_{1} \mathrm{R}$ axis ${ }^{23}$, likely to be associated with insulin resistance ${ }^{24,25}$ but also to severe outcomes of COVID19.

\section{Fermented vegetables}

The fermentation process, a preservation method of the Neolithic age, enabled humans to survive. ${ }^{26}$ Fermented foods are "foods or beverages made via controlled microbial growth (including lactic acid bacteria strains (LABs) and enzymatic conversions of food components." ${ }^{27}$ Many bacteria are used for the fermentation of vegetables but most traditional foods with live bacteria in the low-death rate countries are based on LAB fermentation..$^{5,6,28-30}$

Gut microbiota has an inter-individual variability due to genetic predisposition and diet. ${ }^{31}$ Some foods like cabbage can be fermented by the gut microbiota. ${ }^{32}$

\section{Effects of fermented vegetables on insulin resistance}

Kimchi fermented from many vegetables including cabbage has several effects on insulin resistance associated diseases: anti-diabetic properties ${ }^{33,34}$, cardiovascular diseases ${ }^{35}$, dyslipidemia ${ }^{36}$ or ageing ${ }^{37}$.

Lactobacilli have an inconstant effect on diseases associated with insulin resistance. However, most studies are underpowered or have some methodological flaws. Moreover, not all LAB strains have the same action on insulin resistance. ${ }^{38}$ The redox mechanisms of Lactobacillus spp. are involved in the downregulation of reactive oxygen species (ROS)-forming enzymes and redox stress resistance proteins or genes. ${ }^{39,40} \mathrm{In}$ addition, the nuclear factor erythroid 2-like $2(\mathrm{Nrf-2})$ and nuclear factor kappa B (NF- $\varkappa \mathrm{B})$ are two common transcription factors that also modulate oxidative stress ${ }^{41}$.

There are other products in fermented vegetables that can potentiate the effect of LABs. Kimchi, when fermented for a long time, reduces insulin intolerance to a greater extent than fresh kimchi ${ }^{33}$, indicating that newly formed products during fermentation are important. In particular, Kimchi from cabbage and 
Chinese cabbage contains several glucosinolates ${ }^{1,42,43}$ that can be transformed in sulforaphanes either in the plant itself or by the human microbiome ${ }^{24}$. Sulforaphanes were suggested to be effective in diseases associated with insulin resistance ${ }^{1,44-46}$.

\section{Proposed mechanisms of action of Kimchi and fermented vegetables in COVID 19}

Reactive oxygen species (ROS), such as hydrogen peroxide and superoxide anion, exert beneficial and toxic effects on cellular functions. Nrf2 is a key regulator of the anti-oxidative response. ${ }^{47} \mathrm{Nrf2}$ activity in response to chemical insults is regulated by a thiol-rich protein named KEAP1 (Kelch-like ECH-associated protein 1). The KEAP1-Nrf2 system is the body's dominant defense mechanism against ROS. ${ }^{48}$ Sulforaphane is the most potent activator of $\mathrm{Nrf2}{ }^{3,34}$. "Ancient foods", and particularly those containing Lactobacillus, activate Nrf2. ${ }^{49}$ In different animal models, several strains of Lactobacillus were found to regulate Nrf2. ${ }^{50-52}$ In vitro studies have shown an effect of Lactobacillus on Nrf2. ${ }^{53-55}$

A putative mechanism may be proposed (Figure 2). SARS-CoV-2 downregulates ACE2 inducing an increased insulin resistance associated with oxidative stress through the $\mathrm{AT}_{1} \mathrm{R}$ pathway. Fermented vegetables are often made from cruciferous (Brassica) vegetables that release glucoraphanin converted by the plant or by the gut microbiome into sulforaphane which activates Nrf2 and subsequently reduces insulin intolerance by its potent antioxidant activities. Fermented vegetables contain a high content of Lactobacillus that can activate Nrf2 and impact on the microbiome. Nrf2 may be involved in diseases associated with insulin-resistance ${ }^{24,56-58}$ and may have a role in COVID-19. ${ }^{59}$ Sulforaphane and LABs both have the ability to reduce insulin resistance.

Other putative actions on COVID-19 severity may be postulated. The down-regulation of ACE2 reduces the Ang-1,7 anti-oxidant activity that was found to activate Nrf2. ${ }^{60,61}$ Nrf2 protects against hallmarks of severe COVID-19. It has anti-fibrotic effects on various organs including the lungs, ${ }^{62}$ protects against lung injury and acute respiratory distress syndrome, ${ }^{63}$ and endothelial damage, ${ }^{64}$. Finally, Nrf2 can block IL- 6 in different models of inflammation ${ }^{65}$ and might play a role in the COVID-19 cytokine storm.

These different mechanisms may explain the importance of fermented cabbage in preventing the severity of COVID-19. It is clear that other nutrients, vitamin D and many different foods act on NRF2.

It is not yet known whether sulforaphane and/or LABs may act on the infectivity of SARS-CoV-2. Disulfide bonds can be formed under oxidizing conditions and play an important role in the folding and stability of some proteins. The receptor-binding domain of the viral spike proteins and ACE2 have several cysteine residues. Using molecular dynamics simulations, the binding affinity was significantly impaired when all of the disulfide bonds of both ACE2 and SARS-CoV/CoV-2 spike proteins were reduced to thiol groups. This computational finding possibly provides a molecular basis for the differential COVID-19 cellular recognition due to the oxidative stress. ${ }^{66}$

\section{Kimchi and Asian fermented foods as candidates for the partial control of severe COVID-19}

Mainstream COVID-19 control strategies including social distancing, confinement and intensive case finding, testing, tracing and isolating are so far not enough to provide a SARS-CoV-2-free environment and restore a safe social life. There are hopes for a safe and effective vaccine, but this is unlikely to become available. So, there is a need to explore other potentially useful strategies. An area that has not been sufficiently considered is diet, both as a preventive and/or therapeutically useful intervention, encouraging people to eat more traditional foods containing fermented vegetables. We have suggested that fermented vegetables could be associated with a lower COVID-19 mortality due to their potent antioxidant effect among which sulforaphane and LABs are important. However, many other foods may have a similar activity. It should be noted that dietary supplements that over-activate Nrf2 may have side-effects. ${ }^{67}$

Robust evidence from observational studies would be helpful to formally investigate associations between fermented foods and clinical outcomes in COVID-19. State-of-the-art methods, including the use of DAGs (Directed Acyclic Graphs), may be needed to help assess whether the associations seen are likely to represent causal relationship ${ }^{68}$. A faster approach would be to develop large clinical trials in the appropriate populations. Interventions based on diets with a high intake of fermented foods like Kimchi or other fermented 
foods are unlikely to present ethical difficulties. Furthermore, the fact that a precise mechanism has been proposed would facilitate adding reliable biomarkers to the relevant clinical outcomes. Moreover, new drugs based on the components of these fermented foods may be of interest.

Corresponding author :

Professor Jean Bousquet

CHU Arnaud de Villeneuve, 371 Avenue du Doyen Gaston Giraud, 34295 Montpellier Cedex 5, France Tel +336114288 47, Fax +33467416701

jean.bousquet@orange.fr

Conflict of interest : the authors have no COI to declare.

\section{References}

1. Bousquet J, Anto J, Czarlewski W, et al. Sulforaphane: from death rate heterogeneity in countries to candidate for prevention of severe COVID-19 Allergy 2020;in press.

2. Bousquet J, Anto JM, Iaccarino G, et al. Is diet partly responsible for differences in COVID-19 death rates between and within countries? Clin Transl Allergy 2020;10:16.

3. Fonseca S, Rivas I, Romaguera D, et al. Association between consumption of fermented vegetables and COVID-19 mortality at a country level in Europe MEDRXIV/2020/147025 2020.

4. Fonseca S, Rivas I, Romaguera D, et al. Association between consumption of vegetables and COVID-19 mortality at a country level in Europe. MedRix 2020.

5. Azam M, Mohsin M, Ijaz H, et al. Review - Lactic acid bacteria in traditional fermented Asian foods. Pak J Pharm Sci 2017;30:1803-14.

6. Rhee SJ, Lee JE, Lee CH. Importance of lactic acid bacteria in Asian fermented foods. Microb Cell Fact 2011;10 Suppl 1:S5.

7. Zhang J-j, Dong X, Cao Y-y, et al. Clinical characteristics of 140 patients infected with SARS-CoV-2 in Wuhan, China.n/a.

8. Azkur AK, Akdis M, Azkur D, et al. Immune response to SARS-CoV-2 and mechanisms of immunopathological changes in COVID-19.n/a.

9. Radzikowska U, Ding M, Tan G, et al. Distribution of ACE2, CD147, CD26 and other SARS-CoV-2 associated molecules in tissues and immune cells in health and in asthma, COPD, obesity, hypertension, and COVID-19 risk factors.n/a.

10. Pfaar O, Torres MJ, Akdis CA. COVID-19: A series of important recent clinical and laboratory reports in immunology and pathogenesis of SARS-CoV-2 infection and care of allergy patients.n/a.

11. Du H, Dong X, Zhang J-j, et al. Clinical characteristics of 182 pediatric COVID-19 patients with different severities and allergic status.n/a.

12. Sokolowska M, Lukasik Z, Agache I, et al. Immunology of COVID-19: mechanisms, clinical outcome, diagnostics and perspectives - a report of the European Academy of Allergy and Clinical Immunology (EAACI).n/a.

13. Riggioni C, Comberiati P, Giovannini M, et al. A compendium answering 150 questions on COVID-19 and SARS-CoV-2.n/a.

14. Zabetakis I, Lordan R, Norton C, Tsoupras A. COVID-19: The Inflammation Link and the Role of Nutrition in Potential Mitigation. Nutrients 2020;12. 
15. Finucane FM, Davenport C. Coronavirus and Obesity: Could Insulin Resistance Mediate the Severity of Covid-19 Infection? Front Public Health 2020;8:184.

16. Zhu J, Ji P, Pang J, et al. Clinical characteristics of 3,062 COVID-19 patients: a meta-analysis. J Med Virol 2020.

17. The Novel Coronavirus Pneumonia Emergency Response Epidemiology Team . The epidemiological characteristics of an outbreak of 2019 novel coronavirus diseases (COVID-19) - China, 2020. China CDC Weekly 2020;2:113-22.

18. Argenziano MG, Bruce SL, Slater CL, et al. Characterization and clinical course of 1000 Patients with COVID-19 in New York: retrospective case series. medRxiv 2020.

19. Petrilli CM, Jones SA, Yang J, et al. Factors associated with hospital admission and critical illness among 5279 people with coronavirus disease 2019 in New York City: prospective cohort study. BMJ 2020;369:m1966.

20. Docherty AB, Harrison EM, Green CA, et al. Features of 20133 UK patients in hospital with covid-19 using the ISARIC WHO Clinical Characterisation Protocol: prospective observational cohort study. BMJ 2020;369:m1985.

21. Dalan R, Bornstein SR, El-Armouche A, et al. The ACE-2 in COVID-19: Foe or Friend? Horm Metab Res 2020;52:257-63.

22. Wen H, Gwathmey JK, Xie LH. Oxidative stress-mediated effects of angiotensin II in the cardiovascular system. World J Hypertens 2012;2:34-44.

23. Sarzani R, Giulietti F, Di Pentima C, Giordano P, Spannella F. Disequilibrium between the Classic Renin-Angiotensin System and Its Opposing Arm in Sars-Cov-2 Related Lung Injury. Am J Physiol Lung Cell Mol Physiol 2020.

24. Bousquet J, Anto J, Czarlewski W, et al. Sulforaphane: from death rate heterogeneity in countries to candidate for prevention of severe COVID-19 Allergy 2020;submitted.

25. Ren H, Yang Y, Wang F, et al. Association of the insulin resistance marker TyG index with the severity and mortality of COVID-19. Cardiovasc Diabetol 2020;19:58.

26. Peters A, Krumbholz P, Jager E, et al. Metabolites of lactic acid bacteria present in fermented foods are highly potent agonists of human hydroxycarboxylic acid receptor 3. PLoS Genet 2019;15:e1008145.

27. Marco ML, Heeney D, Binda S, et al. Health benefits of fermented foods: microbiota and beyond. Curr Opin Biotechnol 2017;44:94-102.

28. Patra JK, Das G, Paramithiotis S, Shin HS. Kimchi and Other Widely Consumed Traditional Fermented Foods of Korea: A Review. Front Microbiol 2016;7:1493.

29. Jung JY, Lee SH, Jeon CO. Kimchi microflora: history, current status, and perspectives for industrial kimchi production. Appl Microbiol Biotechnol 2014;98:2385-93.

30. Yoon KY, Woodams EE, Hang YD. Production of probiotic cabbage juice by lactic acid bacteria. Bioresour Technol 2006;97:1427-30.

31. Bibbo S, Ianiro G, Giorgio V, et al. The role of diet on gut microbiota composition. Eur Rev Med Pharmacol Sci 2016;20:4742-9.

32. Tian S, Liu X, Lei P, Zhang X, Shan Y. Microbiota: a mediator to transform glucosinolate precursors in cruciferous vegetables to the active isothiocyanates. J Sci Food Agric 2018;98:1255-60.

33. An SY, Lee MS, Jeon JY, et al. Beneficial effects of fresh and fermented kimchi in prediabetic individuals. Ann Nutr Metab 2013;63:111-9. 
34. Kim EK, An SY, Lee MS, et al. Fermented kimchi reduces body weight and improves metabolic parameters in overweight and obese patients. Nutr Res 2011;31:436-43.

35. Lavefve L, Marasini D, Carbonero F. Microbial Ecology of Fermented Vegetables and Non-Alcoholic Drinks and Current Knowledge on Their Impact on Human Health. Adv Food Nutr Res 2019;87:147-85.

36. Kim SA, Joung H, Shin S. Dietary pattern, dietary total antioxidant capacity, and dyslipidemia in Korean adults. Nutr J 2019;18:37.

37. Das G, Paramithiotis S, Sundaram Sivamaruthi B, et al. Traditional fermented foods with anti-aging effect: A concentric review. Food Res Int 2020;134:109269.

38. Lee E, Jung SR, Lee SY, Lee NK, Paik HD, Lim SI. Lactobacillus plantarum Strain Ln4 Attenuates Diet-Induced Obesity, Insulin Resistance, and Changes in Hepatic mRNA Levels Associated with Glucose and Lipid Metabolism. Nutrients 2018;10.

39. An H, Zhai Z, Yin S, Luo Y, Han B, Hao Y. Coexpression of the superoxide dismutase and the catalase provides remarkable oxidative stress resistance in Lactobacillus rhamnosus. J Agric Food Chem 2011;59:3851-6.

40. Serata M, Iino T, Yasuda E, Sako T. Roles of thioredoxin and thioredoxin reductase in the resistance to oxidative stress in Lactobacillus casei. Microbiology 2012;158:953-62.

41. Kong Y, Olejar KJ, On SLW, Chelikani V. The Potential of Lactobacillus spp. for Modulating Oxidative Stress in the Gastrointestinal Tract. Antioxidants (Basel) 2020;9.

42. Hong E, Kim GH. GC-MS Analysis of the Extracts from Korean Cabbage (Brassica campestris L. ssp. pekinensis) and Its Seed. Prev Nutr Food Sci 2013;18:218-21.

43. Park CH, Yeo HJ, Park SY, Kim JK, Park SU. Comparative Phytochemical Analyses and Metabolic Profiling of Different Phenotypes of Chinese Cabbage (Brassica Rapa ssp. Pekinensis). Foods 2019;8.

44. Xu L, Nagata N, Ota T. Glucoraphanin: a broccoli sprout extract that ameliorates obesity-induced inflammation and insulin resistance. Adipocyte 2018;7:218-25.

45. Teng W, Li Y, Du M, Lei X, Xie S, Ren F. Sulforaphane Prevents Hepatic Insulin Resistance by Blocking Serine Palmitoyltransferase 3-Mediated Ceramide Biosynthesis. Nutrients 2019;11.

46. Sun Y, Zhou S, Guo H, et al. Protective effects of sulforaphane on type 2 diabetes-induced cardiomyopathy via AMPK-mediated activation of lipid metabolic pathways and NRF2 function. Metabolism 2020;102:154002.

47. Vomund S, Schafer A, Parnham MJ, Brune B, von Knethen A. Nrf2, the Master Regulator of AntiOxidative Responses. Int J Mol Sci 2017;18.

48. Yamamoto M, Kensler TW, Motohashi H. The KEAP1-NRF2 System: a Thiol-Based Sensor-Effector Apparatus for Maintaining Redox Homeostasis. Physiol Rev 2018;98:1169-203.

49. Senger DR, Li D, Jaminet SC, Cao S. Activation of the Nrf2 Cell Defense Pathway by Ancient Foods: Disease Prevention by Important Molecules and Microbes Lost from the Modern Western Diet. PLoS One 2016;11:e0148042.

50. Li B, Evivie SE, Lu J, et al. Lactobacillus helveticus KLDS1.8701 alleviates d-galactose-induced aging by regulating Nrf-2 and gut microbiota in mice. Food Funct 2018;9:6586-98.

51. Xu H, Wang J, Cai J, et al. Protective Effect of Lactobacillus rhamnosus GG and its Supernatant against Myocardial Dysfunction in Obese Mice Exposed to Intermittent Hypoxia is Associated with the Activation of Nrf2 Pathway. Int J Biol Sci 2019;15:2471-83. 
52. Qian Y, Zhang J, Zhou X, et al. Lactobacillus plantarum CQPC11 Isolated from Sichuan Pickled Cabbages Antagonizes d-galactose-Induced Oxidation and Aging in Mice. Molecules 2018;23.

53. Xu C, Qiao L, Ma L, et al. Biogenic selenium nanoparticles synthesized by Lactobacillus casei ATCC 393 alleviate intestinal epithelial barrier dysfunction caused by oxidative stress via Nrf2 signaling-mediated mitochondrial pathway. Int J Nanomedicine 2019;14:4491-502.

54. Mu G, Li H, Tuo Y, Gao Y, Zhang Y. Antioxidative effect of Lactobacillus plantarum Y44 on 2,2'azobis(2-methylpropionamidine) dihydrochloride (ABAP)-damaged Caco-2 cells. J Dairy Sci 2019;102:686375 .

55. Kobatake E, Nakagawa H, Seki T, Miyazaki T. Protective effects and functional mechanisms of Lactobacillus gasseri SBT2055 against oxidative stress. PLoS One 2017;12:e0177106.

56. Uruno A, Yagishita Y, Yamamoto M. The Keap1-Nrf2 system and diabetes mellitus. Arch Biochem Biophys 2015;566:76-84.

57. Vasileva LV, Savova MS, Amirova KM, Dinkova-Kostova AT, Georgiev MI. Obesity and NRF2-mediated cytoprotection: Where is the missing link? Pharmacol Res 2020;156:104760.

58. Guo Z, Mo Z. Keap1-Nrf2 signaling pathway in angiogenesis and vascular diseases. J Tissue Eng Regen Med 2020;14:869-83.

59. Hassan SM, Jawad MJ, Ahjel SW, et al. The Nrf2 Activator (DMF) and Covid-19: Is there a Possible Role? Med Arch 2020;74:134-8.

60. Romero A, San Hipolito-Luengo A, Villalobos LA, et al. The angiotensin-(1-7)/Mas receptor axis protects from endothelial cell senescence via klotho and Nrf2 activation. Aging Cell 2019;18:e12913.

61. Cai SM, Yang RQ, Li Y, et al. Angiotensin-(1-7) Improves Liver Fibrosis by Regulating the NLRP3 Inflammasome via Redox Balance Modulation. Antioxid Redox Signal 2016;24:795-812.

62. Liu Q, Gao Y, Ci X. Role of Nrf2 and Its Activators in Respiratory Diseases. Oxid Med Cell Longev 2019;2019:7090534.

63. Zhao H, Eguchi S, Alam A, Ma D. The role of nuclear factor-erythroid 2 related factor 2 (Nrf-2) in the protection against lung injury. Am J Physiol Lung Cell Mol Physiol 2017;312:L155-L62.

64. Chen B, Lu Y, Chen Y, Cheng J. The role of Nrf2 in oxidative stress-induced endothelial injuries. J Endocrinol 2015;225:R83-99.

65. Keleku-Lukwete N, Suzuki M, Yamamoto M. An Overview of the Advantages of KEAP1-NRF2 System Activation During Inflammatory Disease Treatment. Antioxid Redox Signal 2018;29:1746-55.

66. Hati S, Bhattacharyya S. Impact of Thiol-Disulfide Balance on the Binding of Covid-19 Spike Protein with Angiotensin-Converting Enzyme 2 Receptor. ACS Omega 2020;5:16292-8.

67. Smith RE. The Effects of Dietary Supplements that Overactivate the Nrf2/ARE System. Curr Med Chem 2020;27:2077-94.

68. Textor J, van der Zander B, Gilthorpe MS, Liskiewicz M, Ellison GT. Robust causal inference using directed acyclic graphs: the R package 'dagitty'. Int J Epidemiol 2016;45:1887-94.

Figure 1: COVID-19 death rates (Johns Hopkins Coronavirus Resource center) 

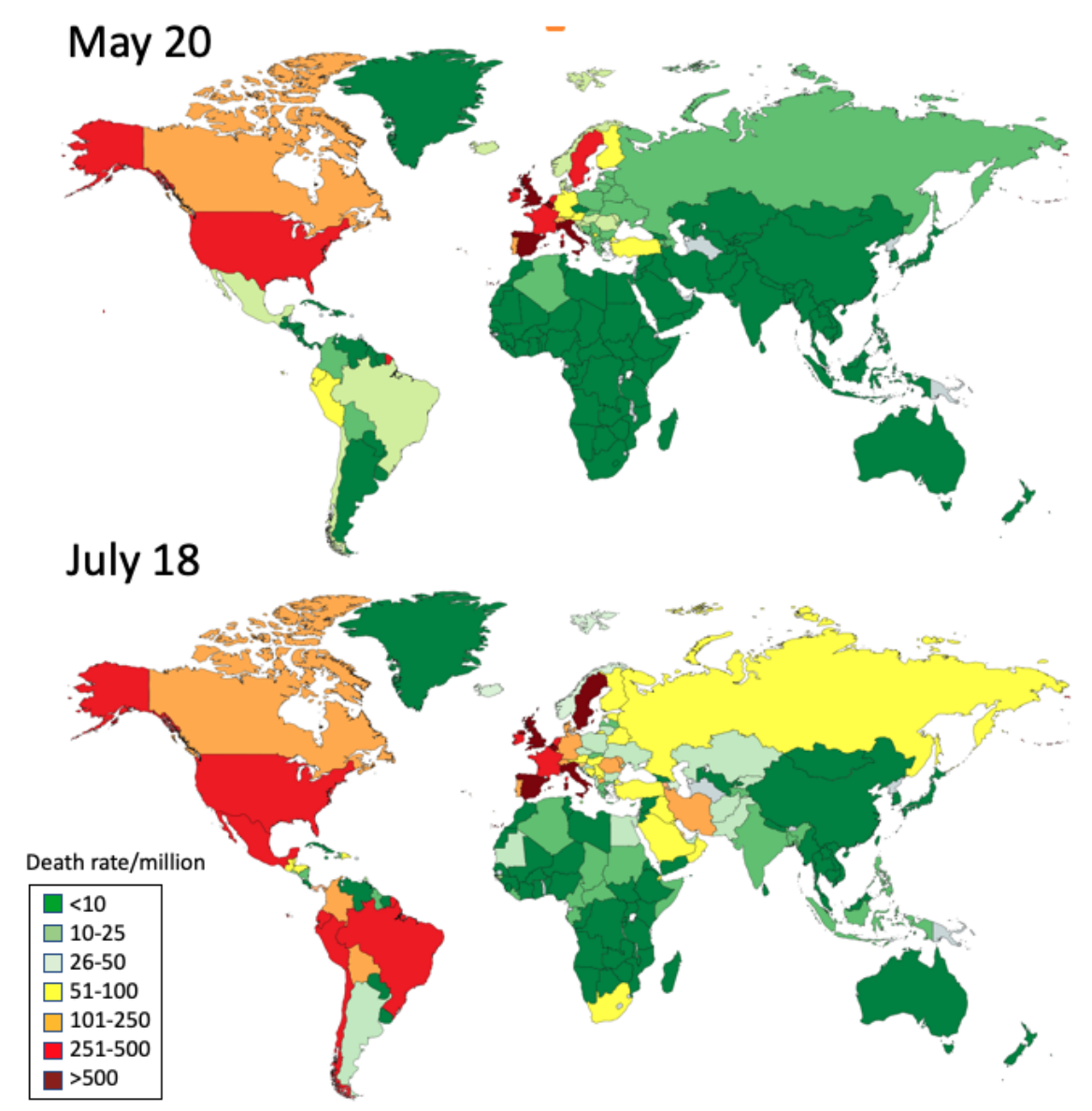

Figure 2: proposed mechanisms of fermented vegetables against COVID-19 (adapted from ${ }^{1}$ )

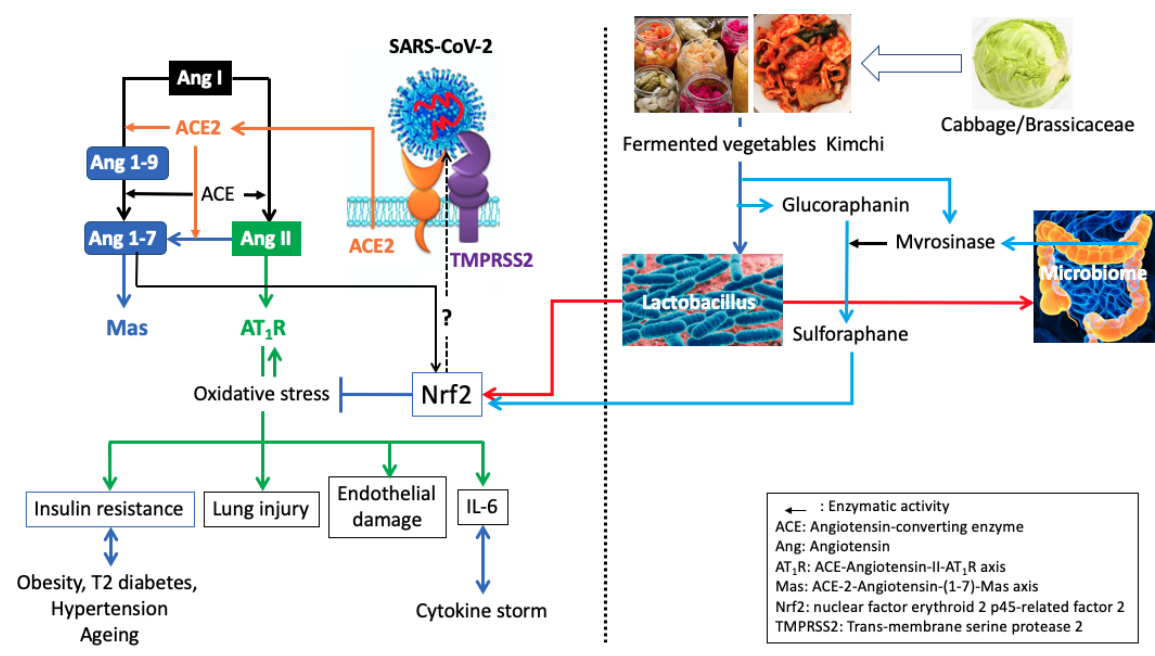

Hosted file

mechanisms of COVID-19 severity.pptx available at https://authorea.com/users/322406/articles/ 
472638-kimchi-and-asian-fermented-vegetables-candidates-for-the-partial-control-of-thecovid-19-pandemic 